\title{
High shear helical flow of a Sisko fluid
}

\author{
Cosimo Chiera $^{1} \quad$ John J. Shepherd ${ }^{2}$
}

(Received 22 December 2011; revised 5 July 2012)

\begin{abstract}
The Sisko fluid model was proposed in response to observed failure of the well-known Bingham model to represent the flows of viscoplastic materials in high shear regimes, such failure increasing with shear rate. We consider the flow of a Sisko fluid between infinitely long coaxial cylinders, when the inner cylinder rotates with constant angular speed and a constant axial fluid flow is maintained. High shear rates are ensured by assuming an inter-cylindrical gap small in relation to the overall geometry. Such a helical flow and geometry is of relevance to a number of applications, including rheometry. We assume a laminar flow and apply a perturbation procedure based on a scaled form of the inter-cylindrical gap dimension as a perturbation parameter to obtain simple explicit approximations for the fluid velocity field as well as the fluid viscosity variation in this gap. We also derive an approximate form of the Reiner-Riwlin equation, linking the defining fluid parameters to measurable quantities in the flow.
\end{abstract}

http://journal.austms.org.au/ojs/index.php/ANZIAMJ/article/view/5008 gives this article, (c) Austral. Mathematical Soc. 2012. Published July 17, 2012. ISSN 1446-8735. (Print two pages per sheet of paper.) Copies of this article must not be made otherwise available on the internet; instead link directly to this URL for this article. 


\section{Contents}

1 Introduction

C405

2 Governing equations

C406

3 Perturbation analysis for flow in a narrow gap

C410

4 Discussion

C414

References

C420

\section{Introduction}

Helical flows occur when fluid in the gap between infinitely long coaxial cylinders has an axial fluid velocity superimposed on a transverse rotational motion. Such flows are of interest in applications, where they arise in the modelling of the action of a cup and bob rheometer modified to allow axial flow in order to carry out measurements on slurries and other settling mixtures (Chiera, Connell and Shepherd [3]).

When the fluid is incompressible and Newtonian, so that the stress and rate of shearing are linearly related, such helical flow is well understood and solutions of the flow problem are well-represented [7, Ch. IV]. However, when the fluid is incompressible, with the stress a nonlinear function of the rate of shearing alone; that is, it is a generalized Newtonian fluid (a particular class of non-Newtonian fluids) [1], the problem of obtaining the velocity profile and associated fluid properties is considerably more difficult and numerical solution techniques usually must be employed. While Coleman and Noll [5] solved this problem in general terms for all such fluids, their analysis converted a nonlinear boundary value problem to a nonlinear algebraic one, leaving the details of the solution of either incomplete. This phenomenon is clearly 
illustrated in the analysis of the helical flow of a power law fluid [1, p. 148], a generalized Newtonian fluid.

For the rheometer application above, the inter-cylindrical gap is kept small, to minimize experimental end effects. This is significant for the following analysis in that a perturbation procedure based on some normalized form of the inter-cylindrical gap as perturbation parameter is used to solve the nonlinear equations of motion. It also means that the flow in the intercylindrical gap experiences high shear rates; that is, the flow is a high shear flow. This approach has been used to analyse such helical flow of similar generalized Newtonian fluids [3, 6].

We apply a perturbation approach to the laminar helical flow of a Sisko fluid, a generalized Newtonian fluid model used to represent viscous inelastic shear thinning fluids [8], such as greases at high shear rates. This model was seen to be an improvement on earlier models of these materials. Our analysis is based upon the work of Chiera [2], uses arguments analogous to those of Farrugia, Shepherd and Stacey [6] for the Powell-Eyring fluid, to parallel and extend the earlier work of Chiera, Connell and Shepherd [4].

\section{Governing equations}

We consider steady helical flow of an incompressible viscous fluid in the infinitely long annular region described in cylindrical polars $(r, \theta, z)$ by $R_{1} \leqslant$ $r \leqslant R_{2}, 0 \leqslant \theta \leqslant 2 \pi,-\infty \leqslant z \leqslant \infty$. The inner cylindrical surface $r=R_{1}$ is given a constant angular velocity $\Omega>0$, the outer cylinder $r=R_{2}$ is held stationary, and a given axial flow rate $Q>0$ is imposed.

The equations of motion for such a flow are well documented $[5,6]$. With the $z$-axis taken as vertically down and assuming symmetry about this axis, the velocity field takes the form

$$
\left(u_{r}, u_{\theta}, u_{z}\right)=(0, r W(r), v(r))
$$


for appropriate functions $V(r)$ and $W(r)$, while the momentum equations yield the differential equations

$$
H V^{\prime}(r)=\alpha r+\frac{\beta}{r}, \quad H W^{\prime}(r)=-\frac{M}{2 \pi r^{3}},
$$

together with the boundary conditions

$$
\mathrm{V}\left(\mathrm{R}_{1}\right)=\mathrm{V}\left(\mathrm{R}_{2}\right)=0, \quad \mathrm{~W}\left(\mathrm{R}_{1}\right)=\Omega, \quad \mathrm{W}\left(\mathrm{R}_{2}\right)=0 .
$$

In (1) $\mathrm{H}$ is the fluid viscosity, $M>0$ is the moment per unit length exerted on the inner cylinder $r=R_{1}$, and $\alpha$ and $\beta$ are constants to be determined by the application of the boundary conditions. Further, the differential equations (1) yield the relationship

$$
V^{\prime}(r)=-2 \pi\left(\alpha r^{2}+\beta\right) r^{2} W^{\prime}(r) / M .
$$

The volume flow rate

$$
\mathrm{Q}=2 \pi \int_{\mathrm{R}_{1}}^{\mathrm{R}_{2}} r V(r) d r
$$

( $\mathrm{Q}$ is regarded as known, so that (4) is a constraint on $\mathrm{V}(\mathrm{r})$ ).

The local rate of shearing,

$$
\mathrm{K}=\sqrt{\left[\left(\mathrm{rW} \mathrm{W}^{\prime}\right)^{2}+\mathrm{V}^{\prime 2}\right] / 2}
$$

in terms of the gradients of $\mathrm{V}$ and $\mathrm{W}$.

The boundary conditions (2) imply that $W^{\prime}(r)<0$ in all $R_{1}<r<R_{2}$; and applying (3) to (5) converts the local rate of shearing to

$$
K=-r W^{\prime} \phi(r, \alpha, \beta) / \sqrt{2}, \quad \phi(r, \alpha, \beta)=\sqrt{1+(2 \pi / M)^{2} r^{2}\left(\alpha r^{2}+\beta\right)^{2}} .
$$

As noted, for generalized Newtonian fluids, $\mathrm{H}$ is a function of $\mathrm{K}$ alone; that is, $\mathrm{H}=\mathrm{H}(\mathrm{K})$. For the Sisko fluid, this relationship is

$$
H(K)=H_{0}\left(1+\left(\frac{K}{K_{0}}\right)^{n-1}\right)
$$


where $\mathrm{H}_{0}, \mathrm{~K}_{0}$ and $n$ are positive constants, with $0 \leqslant n<1$.

In many cases, the range $0 \leqslant n \leqslant 1$ is used. Putting $n=1$ in (7) gives $\mathrm{H}(\mathrm{K})=2 \mathrm{H}_{0}$, and the Sisko fluid reduces to a Newtonian one, of constant viscosity $2 \mathrm{H}_{0}$. However, when $n=1$, there is no variation of $H$ with $K$; and since it is the aim of this article to analyze the flow when $\mathrm{H}$ varies with $\mathrm{K}$, especially as $\mathrm{K} \rightarrow \infty$, we exclude the value $\mathrm{n}=1$. Moreover, the Newtonian flow case may be solved exactly [7]. For $0 \leqslant n<1, H(K) \rightarrow H_{0}$, a constant, as $\mathrm{K} \rightarrow \infty$; that is, the Sisko fluid tends to the Newtonian at high shear rates. Thus, it could be classed as a limiting Newtonian fluid.

The second equation in (1) gives, from (6) and (7),

$$
\left(\frac{-r W^{\prime} \phi(r, \alpha, \beta}{\sqrt{2} K_{0}}\right)^{n-1}=-\frac{M}{2 \pi \mathrm{H}_{0} r^{3} W^{\prime}}-1,
$$

which is a first order nonlinear differential equation for $\mathrm{W}$ that can be solved, in principle, subject to one of the boundary conditions (2).

The problem (1)-(8) is converted to dimensionless form by defining the radial distance $R=\left(R_{1}+R_{2}\right) / 2$ and the dimensionless inter-cylindrical gap width $\epsilon=\left(R_{2}-R_{1}\right) /\left(R_{1}+R_{2}\right)$. Thus small values of $\epsilon$ correspond to a small inter-cylindrical gap.

Dimensionless constants $\mathrm{a}, \mathrm{b}, \sigma$ and radial variable $s$ are then defined by

$$
a=-2 \pi \epsilon R^{3} \alpha / M, \quad b=2 \pi \epsilon R \beta / M, \quad \sigma^{2}=b / a, \quad r=R s,
$$

respectively, while dimensionless forms of $\mathrm{V}, \mathrm{W}, \mathrm{K}$ and $\mathrm{H}$, denoted by $v(\mathrm{~s})$, $w(s), \kappa(s)$ and $\eta(s)$, are defined by

$$
v=\Delta \mathrm{V}(\mathrm{Rs}), \quad w=\Delta \mathrm{RW}(\mathrm{Rs}), \quad \mathrm{k}=\sqrt{2} \Delta \mathrm{RK}(\mathrm{Rs}), \quad \eta=\mathrm{H}(\mathrm{K}(\mathrm{Rs})) / \mathrm{H}_{0},
$$

where $\Delta=2 \pi \mathrm{H}_{0} \mathrm{R} /(M \epsilon)$.

Finally, define dimensionless parameters

$$
\gamma=\left(\frac{M \epsilon}{2 \sqrt{2} \pi \mathrm{H}_{0} \mathrm{~K}_{0} \mathrm{R}^{2}}\right)^{\mathrm{n}-1}, \quad \mathrm{q}=\frac{2 \mathrm{H}_{0} \mathrm{Q}}{M \epsilon^{2} \mathrm{R}}, \quad \omega=\frac{2 \pi \mathrm{H}_{0} \mathrm{R}^{2} \Omega}{M \epsilon} .
$$


Parameter $\gamma$ derives from the fluid parameters $H_{0}, K_{0}$ and $n$, while $q$ and $\omega$ are related to the axial flow rate $\mathrm{Q}$ and inner cylinder angular velocity $\Omega$, respectively.

The dimensionless flow problem in the inter-cylindrical gap, $s_{1}=1-\epsilon \leqslant s \leqslant$ $1+\epsilon=s_{2}$, now consists of the nonlinear differential equation for $w(s)$,

$$
\left(-s \mathcal{W}^{\prime} \psi\right)^{\mathrm{n}-1} \gamma=-\frac{1}{\mathrm{~s}^{3} \in \mathcal{W}^{\prime}}-1
$$

where $\psi(s, a, \sigma)=\sqrt{1+(s a / \epsilon)^{2}\left(s^{2}-\sigma^{2}\right)^{2}}$, with the linked equation for $v(s)$

$$
v^{\prime}=a s^{2}\left(s^{2}-\sigma^{2}\right) w^{\prime} / \epsilon,
$$

subject to the boundary conditions

$$
v\left(s_{1}\right)=v\left(s_{2}\right)=0, \quad w\left(s_{1}\right)=w, \quad w\left(s_{2}\right)=0 .
$$

The flow condition (4) becomes, on integrating by parts, and using the first set of boundary conditions in (11),

$$
\int_{s_{1}}^{s_{2}}\left(s^{2}-\sigma^{2}\right) v^{\prime}(s) d s=-\epsilon q
$$

while $\mathrm{K}$ and $\mathrm{H}(\mathrm{K})$ take the dimensionless forms

$$
\kappa(s)=-s w^{\prime}(s) \psi(s, a, \sigma), \quad \eta(\kappa)=1+\gamma \kappa^{n-1} .
$$

Given a, $\sigma, \gamma, \epsilon$ and $\mathbf{q}$, the differential equations (9) and (10) are then integrated subject to any $v-w$ pair of the boundary conditions (11), to obtain $v(s)$ and $w(s)$. The full set of conditions (11), with the flow condition (12) yield three nonlinear equations

$$
f_{i}(a, \sigma, \gamma, \omega, q, \epsilon)=0, \quad i=1,2,3 .
$$

Any two of these may be solved, in principle, for a and $\sigma$ in terms of the other quantities; and, applying these values to the third equation

$$
\mathrm{F}(\gamma, \omega, \mathrm{q}, \epsilon)=0 \text {. }
$$


This equation defines a relationship linking $\gamma$ and $\omega$, with $\mathbf{q}$ and $\epsilon$ present as parameters, which is equivalent to an analogous relationship linking $\Omega$ to $M$. This relationship, termed the Reiner-Riwlin equation, is fundamental to this flow for the given fluid [1, p. 231]. If $\gamma$ is assumed given (that is, the flow occurs under constant torque conditions - typical of rheometer applications), this relationship determines $\omega$ in terms of $q$ and $\epsilon$. Thus, the quantities $a$ and $\sigma$ are viewed as functions of $\mathbf{q}$ and $\epsilon$. Consequently, the functions $w$ and $v$ described above are regarded as functions of $\mathrm{q}$ and $\epsilon$ as well as $\mathrm{s}$.

In general, the nonlinearity of the component equations above renders explicit calculation of the Reiner-Riwlin equation well-nigh impossible. Thus, the solution of the problem (9)-(12), and formulation of the Reiner-Riwlin equation must be carried out numerically. This task is complex and is beyond the scope of the present article. However, in one case of interest in applications, solutions are obtained by approximate methods. For a large class of helical flows, the inter-cylindrical gap is small, and thus so is $\epsilon$ (values of $R_{1}=0.024$ and $R_{2}=0.025$, as used in the laboratory model for the rheometer application [3], give $\epsilon=0.0204$ ), while the $\epsilon$-dependence of $q$ is predictable; so that perturbation methods based on $\epsilon \rightarrow 0$ apply.

\section{Perturbation analysis for flow in a narrow gap}

We apply a perturbation procedure based on the limit $\epsilon \rightarrow 0$ (corresponding to a small inter-cylindrical gap) to the problem defined by (9)-(12), to obtain approximate expressions for $v(s)$ and $w(s)$ and the constants $a$ and $\sigma$. This requires estimating the order of terms in (9)-(12). Simple arguments based on the physical situation and geometry [6] lead us to estimate all of $a, b$, $\sigma, \gamma, q, \omega$ as $\mathcal{O}(1)$ constants, while $\mathcal{v}(\mathrm{s}), \boldsymbol{w}(\mathrm{s})$ and $\psi(\mathrm{s}, \mathrm{a}, \sigma)$ are $\mathcal{O}(1)$ functions, and $\mathcal{v}^{\prime}(\mathrm{s})$ and $\boldsymbol{w}^{\prime}(\mathrm{s})$ are $\mathcal{O}\left(\epsilon^{-1}\right)$ functions. In view of these estimates, $\kappa(s)$ is $\mathcal{O}\left(\epsilon^{-1}\right)$. Thus, small $\epsilon$ values correspond to high shear flow in the 
(narrow) inter-cylindrical gap.

The unknowns in this problem are the functions $w(s)$ and $v(s)$, and the constants $a$ and $\sigma$. We now propose that these be represented as perturbation expansions in terms of the (small) parameter $\epsilon$ that, based on the estimates above, take the form

$$
\begin{aligned}
a(\epsilon) & \sim a_{0}+\mu_{1}(\epsilon) a_{1}+\mu_{2}(\epsilon) a_{2}+\cdots, \\
\sigma(\epsilon) & \sim \sigma_{0}+\delta_{1}(\epsilon) \sigma_{1}+\delta_{2}(\epsilon) \sigma_{2}+\cdots, \\
\epsilon W^{\prime}(s) & \sim u_{0}(s)+v_{1}(\epsilon) u_{1}(s)+v_{2}(\epsilon) u_{2}(s)+\cdots, \\
v^{\prime}(s) & \sim v_{0}^{\prime}(s)+\xi_{1}(\epsilon) v_{1}^{\prime}(s)+\xi_{2}(\epsilon) v_{2}^{\prime}(s)+\cdots,
\end{aligned}
$$

where $\mu_{i}(\epsilon), \delta_{i}(\epsilon), v_{i}(\epsilon)$ and $\xi_{i}(\epsilon)$ are asymptotic sequences of gauge functions of $\epsilon$ as $\epsilon \rightarrow 0$; that is, $\mu_{i+1} / \mu_{i} \rightarrow 0$ as $\epsilon \rightarrow 0$. Since $w^{\prime}(s)=\mathcal{O}\left(\epsilon^{-1}\right)$, the expansion (16), which is the expansion for $\epsilon \mathcal{W}^{\prime}$, is an $\mathcal{O}(1)$ quantity.

We substitute (14)-(17) into the equations (9)-(10) and (11)-(12) and on seeking a balance of orders of terms as $\epsilon \rightarrow 0$, obtain expressions for the constants $\mu_{1}(\epsilon), \delta_{1}(\epsilon), v_{1}(\epsilon)$ and $\xi_{1}(\epsilon)$, as well as the functions $u_{0}(s), u_{1}(s)$, $v_{0}^{\prime}(s)$ and $v_{1}^{\prime}(s)$. Integration of these last, subject to the boundary conditions (11) at the outer boundary $s=s_{2}$, then yield expressions for $w_{0}(s)$, $w_{1}(s), v_{0}(s)$ and $v_{1}(s)$, and hence via (16) and (17) two term approximations to $w(s)$ and $v(s)$ that satisfy the boundary conditions (11) at $s=s_{2}$.

Making the replacement $w^{\prime}=\epsilon^{-1} \in \mathcal{W}^{\prime}$, then substituting (14), (15) and (16) into (9) gives, to leading order

$$
\epsilon^{n-1}\left[-s u_{0}+\cdots \psi\left(s, a_{0}+\cdots, \sigma_{0}+\cdots\right)\right]^{n-1} \gamma=-\frac{1}{s^{3} u_{0}+\cdots}-1 .
$$

From equation (18), equating leading order terms gives

$$
u_{0}=-\frac{1}{s^{3}}
$$

which corresponds to the Newtonian description of fluid viscosity. This is to be expected, since as $\epsilon \rightarrow 0$, the shear rate $\kappa \rightarrow \infty$, and the Sisko fluid tends to Newtonian. 
Equating higher order terms in (18) gives, on applying (19),

$$
-\frac{u_{1}}{s^{3} u_{0}^{2}} v_{1}(\epsilon)+\cdots=\gamma\left(\frac{-s u_{0} \psi_{0}}{\epsilon}\right)^{n-1}
$$

where we define

$$
\psi_{0}=\psi\left(s, a_{0}, \sigma_{0}\right)=\left(1+\left(\frac{a_{0} s}{\epsilon}\right)^{2}\left(s^{2}-\sigma_{0}^{2}\right)^{2}\right)^{1 / 2} .
$$

Choosing $\vee_{1}(\epsilon) \equiv \epsilon^{1-n}$ gives, from (19) and (20),

$$
u_{1}=\gamma s^{-2 n-1} \psi_{0}^{n-1}
$$

Similarly, substituting the perturbation expansions (14), (15), (16), and (19) into (10) gives, on considering leading order terms,

$$
v_{0}^{\prime}=\frac{a_{0}}{\epsilon^{2}}\left(s^{2}-\sigma_{0}^{2}\right) s^{-1} .
$$

Since $s^{2}-\sigma_{0}^{2}$ is $\mathcal{O}(\epsilon), v_{0}^{\prime}(s)$ is an $\mathcal{O}\left(\epsilon^{-1}\right)$ quantity, as required. Futher, repeating the process for generating $v_{0}^{\prime}(\mathrm{s})$ gives $v_{1}^{\prime}(\mathrm{s})$ as

$$
\begin{aligned}
v_{1}^{\prime}(s) \xi_{1}(\epsilon)= & \left(\frac{s}{\epsilon}\right)^{2}\left[\left(a_{0} u_{1}\left(s^{2}-\sigma_{0}^{2}\right) \epsilon^{1-n}\right)-2 a_{0} \sigma_{0} \sigma_{1} u_{0} \delta_{1}(\epsilon)\right. \\
& \left.+a_{1} u_{0}\left(s^{2}-\sigma_{0}^{2}\right) \mu_{1}(\epsilon)\right] .
\end{aligned}
$$

Since we require that all the terms shown in (24) are to be included in $v_{1}^{\prime}(\mathrm{s})$, then we choose the gauge functions

$$
\xi_{1}(\epsilon) \equiv \epsilon^{-n}, \quad \delta_{1}(\epsilon) \equiv \epsilon^{2-n}, \quad \mu_{1}(\epsilon) \equiv \epsilon^{1-n} .
$$

Thus, on substituting for $\boldsymbol{u}_{0}$ and $\boldsymbol{u}_{1}$, as given in (19) and (22),

$$
v_{1}^{\prime}(s)=\left[2 a_{0} \sigma_{0} \sigma_{1}-\frac{a_{1}}{\epsilon}\left(s^{2}-\sigma_{0}^{2}\right)\right] s^{-1}-\frac{a_{0} \gamma}{\epsilon}\left(s^{2}-\sigma_{0}^{2}\right) s^{-2 n+1} \psi^{n-1} .
$$


Having now determined the gauge functions $\mu_{1}(\epsilon), \delta_{1}(\epsilon), v_{1}(\epsilon)$ and $\xi_{1}(\epsilon)$ it remains to determine the coefficients $a_{0}, a_{1}, \sigma_{0}$ and $\sigma_{1}$. This is done by using the expressions for $v_{0}^{\prime}(\mathrm{s})$ and $v_{1}^{\prime}(\mathrm{s})$, in combination with the boundary conditions (11) and the flow condition (12), to generate the coefficient pairs $a_{0}, \sigma_{0}$ and $a_{1}, \sigma_{1}$.

Integrating $v_{0}^{\prime}(s)$ (given in (23)) subject to the boundary conditions (11) gives

$$
\sigma_{0}=\sqrt{\frac{\frac{1}{2}\left(s_{2}^{2}-s_{1}^{2}\right)}{\ln \left(s_{2} / s_{1}\right)}}=1+\mathcal{O}\left(\epsilon^{2}\right),
$$

on noting that $s_{1}=1-\epsilon$ and $s_{2}=1+\epsilon$.

Similarly, using $v_{0}^{\prime}(s)$ in the equation linking the scaled axial flow rate $\mathbf{q}$ and the scaled axial velocity field component (12) yields

$$
a_{0}=\frac{\epsilon^{3} q}{\int_{s_{1}}^{s_{2}} s^{-1}\left(s^{2}-\sigma_{0}^{2}\right)^{2} d s}=\frac{3}{8} q+\mathcal{O}\left(\epsilon^{2}\right),
$$

The values for $\sigma_{0}$ and $a_{0}$ are those obtained for the Newtonian fluid.

Using the same method as was used to generate (27) and (28) yields, after some computation

$$
a_{1}=a_{0} \gamma D\left(\ln \left(I_{3} \frac{s_{2}}{s_{1}}\right)-I_{1} H_{2}\right)
$$

where

$$
\begin{aligned}
D & =\int_{s_{1}}^{s_{2}} s^{-1}\left(s^{2}-\sigma_{0}^{2}\right) d s \int_{s_{1}}^{s_{2}} s d s-\int_{s_{1}}^{s_{2}} s\left(s^{2}-\sigma_{0}^{2}\right) d s \int_{s_{1}}^{s_{2}} s^{-1} d s \\
& =-\frac{16}{3} \epsilon^{3}+\mathcal{O}\left(\epsilon^{5}\right), \\
H_{p} & =\frac{1}{p}\left(s_{2}^{p}-s_{1}^{p}\right), \\
I_{p} & =\int_{s_{1}}^{s_{2}} s^{-2 n+p}\left(s^{2}-\sigma_{0}^{2}\right) \psi_{0}^{n-1} d s .
\end{aligned}
$$


Similarly,

$$
\sigma_{1}=\frac{\gamma}{2 \sigma_{0} \epsilon \mathrm{D}}\left\{\mathrm{I}_{3}\left[\mathrm{H}_{2}-\sigma_{0}^{2} \ln \left(\mathrm{s}_{2} / \mathrm{s}_{1}\right)\right]-\mathrm{I}_{1}\left[\mathrm{H}_{4}-\sigma_{0}^{2} \mathrm{H}_{2}\right]\right\} .
$$

Thus the scaled axial velocity field component

$$
\begin{aligned}
v(s, \epsilon)= & \frac{a_{0}}{\epsilon^{2}} \int_{s}^{s_{2}} \frac{t^{2}-1}{t} d t-\epsilon^{-n} \int_{s}^{s_{2}}\left[\left(2 a_{0} \sigma_{0} \sigma_{1}-\frac{a_{1}}{\epsilon}\left(t^{2}-\sigma_{0}^{2}\right)\right) t^{-1}\right. \\
& \left.-\frac{a_{0} \gamma}{\epsilon}\left(t^{2}-\sigma_{0}^{2}\right) t^{-2 n+1} \psi_{0}^{n-1}\right] d t+\cdots,
\end{aligned}
$$

while the scaled transverse velocity

$$
w(s, \epsilon)=\epsilon^{-1} \int_{s}^{s_{2}} t^{-3} d t+\epsilon^{-n} \gamma \int_{s}^{s_{2}} t^{-2 n+1} \psi_{0}^{2(n-1)} d t+\cdots .
$$

\section{Discussion}

The expressions (34) and (35) are readily evaluated approximations to the functions $(w(s), v(s))$ and the constants a and $\sigma$ for small $\epsilon$. They may be used to obtain approximate expressions for the significant features of this high shear helical flow. Each consists of a leading order term corresponding to a Newtonian flow field, followed by a higher order correction involving the parameter $\gamma$, which incorporates the Sisko parameters $\mathrm{H}_{0}, \mathrm{~K}_{0}$ and $\mathrm{n}$.

If the third of the boundary conditions (11) is applied to (35), we obtain

$$
\omega_{0}=\epsilon^{-1} \int_{s_{1}}^{s_{2}} t^{-3} d t+\epsilon^{-n} \gamma \int_{s_{1}}^{s_{2}} t^{-2 n+1} \psi_{0}^{2(n-1)} d t+\cdots
$$

Equation (36) is a dimensionless approximate form of the Reiner-Riwlin equation, which links the (dimensionless) angular speed of rotation, $\omega_{0}$, of the central cylinder to the Sisko parameters $\mathrm{H}_{0}, \mathrm{~K}_{0}$ and $\mathrm{n}$ incorporated into the dimensionless parameter $\gamma$. This constitutes an approximate constraint 
on the parameters defining the helical flow. If this in rewritten in the original dimensional variables, it provides a link between the parameters $\mathrm{H}_{0}, \mathrm{~K}_{0}$ and $n$ and the observable quantities $\mathrm{M}, \mathrm{Q}$ and $\Omega$. Experimental readings of the latter may then be used to calculate the Sisko parameters $\mathrm{H}_{0}, \mathrm{~K}_{0}$ and $\mathrm{n}$.

The approximations (34) and (35) are used to represent the fluid velocity field $(s w(s), v(s))$ in the inter-cylindrical gap.

Thus Figure 1 shows the axial velocity profile $v(\mathrm{~s})$ as represented by these approximations, for several values of $\gamma$, the 'Sisko parameter', indicating increasing levels of non-Newtonian behaviour. It is apparent that as $\gamma$ increases from zero, corresponding to Newtonian flow, the axial velocity increases throughout the gap, roughly linearly. There is also no discernible shift in the location of the velocity peak, as might be expected from the estimate $\sigma=1+\mathcal{O}\left(\epsilon^{2-n}\right)$. Figure 2 shows the transverse velocity profile $\epsilon \mathcal{W}(s)$ for the same $\gamma$ values. Here the overall transverse velocity increases with $\gamma$ throughout the gap. In particular, the transverse velocity $s_{1} \omega$ at the inner cylinder increases with $\gamma$, reminding us that the value of $\omega$ is not arbitrarily prescribed, but is linked to the other fluid parameters through the Reiner-Riwlin equation.

The approximations (34) and (35) are also used to obtain approximate expressions for the rate of shearing $k(s)$ and the viscosity profile $\eta(s)$ as functions of $s$. These are shown in Figures 3 and 4 respectively.

Figure 3 shows the rate of shear profile $\mathrm{k}(\mathrm{s})$ obtained by applying (34) and (35) to the first equation in (13), for the same $\gamma$ values. In all cases, the rate of shearing has a minimum near the centre of the inter-cylindrical gap, but increases overall with $\gamma$. The minimum location shifts towards the inner cylinder as $\gamma$ increases.

Figure 4 shows the viscosity profile $\eta(s)$ using (34) and (35) in (13) for the same $\gamma$ values. As expected, the viscosity is constant when $\gamma=0$, but shows (limited) variation for nonzero values. As $\gamma$ increases, a maximum viscosity evolves near the gap centreline. 


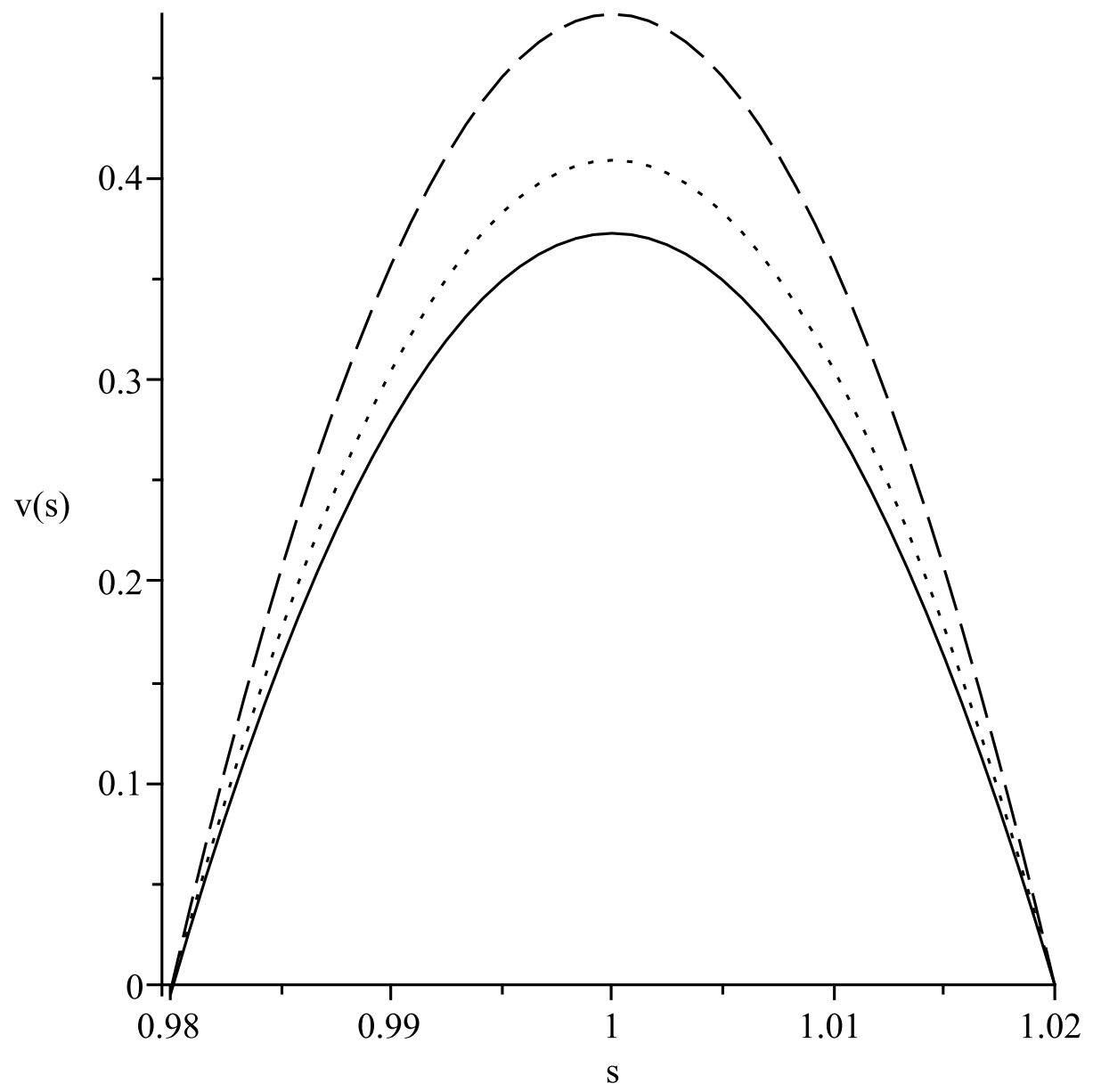

Figure 1: Dimensionless axial velocity profile $v(\mathrm{~s})$ across the inter-cylindrical gap as given by the approximation (34) for $\epsilon=0.02, \gamma=0$ (solid curve), $\gamma=1$ (dotted curve), $\gamma=3$ (dashed curve). 


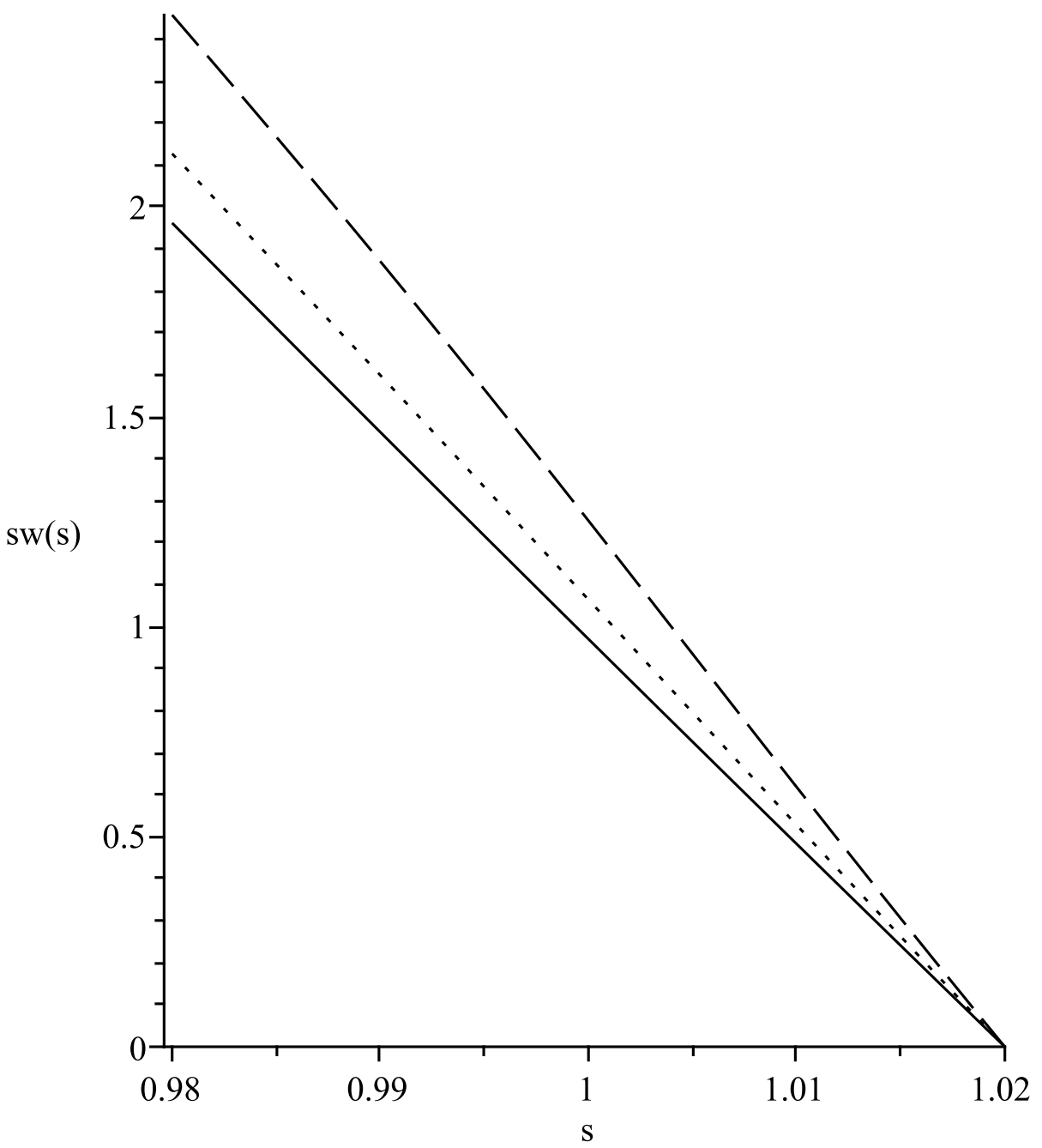

Figure 2: Dimensionless transverse velocity profile $s w(s)$ across the intercylindrical gap as given by the approximation (35) for $\epsilon=0.02, \gamma=0$ (solid curve), $\gamma=1$ (dotted curve), $\gamma=3$ (dashed curve). 


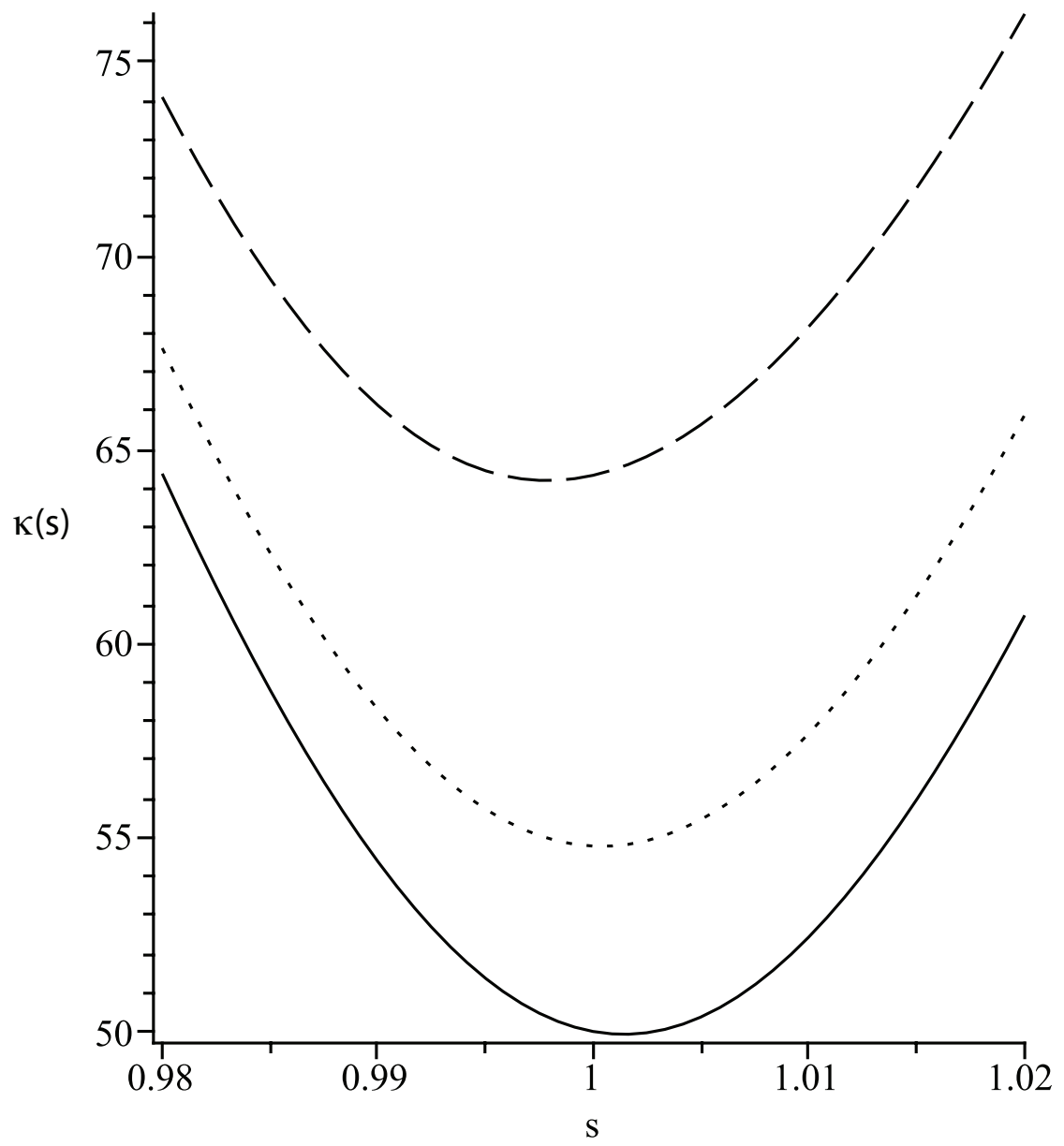

Figure 3: Dimensionless rate of shearing profile $\mathrm{k}(\mathrm{s})$ across the inter-cylindrical gap as given by the approximations (34), (35) to (13) for $\epsilon=0.02, \gamma=0$ (solid curve), $\gamma=1$ (dotted curve), $\gamma=3$ (dashed curve). 


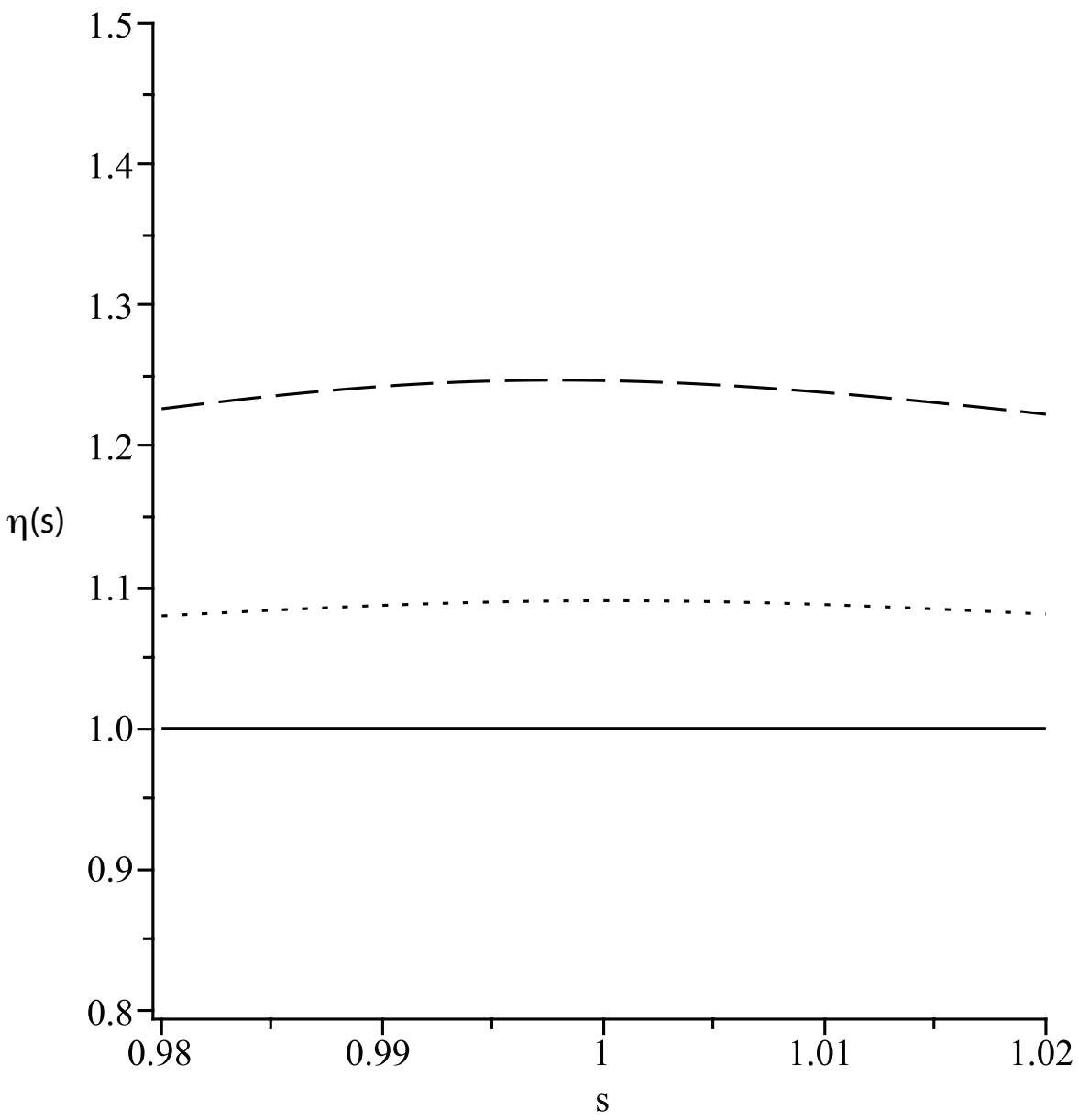

Figure 4: Dimensionless rate of viscosity profile $\eta(s)$ across the intercylindrical gap as given by the approximations (34), (35) to (13) for $\epsilon=0.02$, $\gamma=0$ (solid curve), $\gamma=1$ (dotted curve), $\gamma=3$ (dashed curve). 


\section{References}

[1] R. B. Bird, R. C. Armstrong and D. Hassager, Dynamics of Polymetric liquids, Vol. 1, John Wiley and Sons, 1987. C405, C406, C410

[2] C. Chiera, Perturbation Analysis of the Helical Flow of Generalized Newtonian Fluids, Ph.D. Thesis, RMIT, 2004 C406

[3] C. Chiera, H. J. Connell and J. J. Shepherd, Perturbation analysis of the helical flow of non-Newtonian fluids with application to a recirculating coaxial cylinder rheometer, Mathematical and Computer Modelling, 18, No 10: 131-140, 1993. doi:10.1016/0895-7177(93)90222-K C405, C406, C410

[4] C. Chiera, H. J. Connell and J. J. Shepherd, On the helical flow of a Sisko model fluid, Proc. 2nd Pacific Rim Conf. Rheology (PRCR2), Melbourne, Australia, 33-34, July 1997 C406

[5] B. D. Coleman and W. Noll, Helical flow of General Fluids, J. App. Phys., 10: 1508-1512, 1959. doi:10.1063/1.1734990 C405, C406

[6] M. T. Farrugia, J. J. Shepherd and A. J. Stacey, A perturbation analysis of the flow of a Powell-Eyring fluid between coaxial cylinders, ANZIAM J., 52 257-270, 2011. http://journal.austms.org.au/ojs/ index.php/ANZIAMJ/article/view/3748 C406, C410

[7] W. E. Langlois, Slow Viscous Flow, Macmillan, New York, 1964 C405, C408

[8] A. W. Sisko, The flow of lubricating greases, Ind. \&5 Eng. Chem., 50 (12), 1789-1792, 1958

http://pubs.acs.org/doi/abs/10.1021/ie50588a042 C406 


\section{Author addresses}

\section{Cosimo Chiera,}

mailto: cos@AussieBattlerSites.com.au

2. John J. Shepherd, RMIT University

mailto: jshep@rmit.edu.au 